\title{
Stability and Hopf Bifurcation of a Computer Virus Model with Infection Delay and Recovery Delay
}

\author{
Haitao Song, ${ }^{1}$ Qiaochu Wang, ${ }^{2}$ and Weihua Jiang' \\ ${ }^{1}$ Department of Mathematics, Harbin Institute of Technology, Harbin 150001, China \\ ${ }^{2}$ School of Architecture, Harbin Institute of Technology, Harbin 150001, China \\ Correspondence should be addressed to Weihua Jiang; jiangwh@hit.edu.cn
}

Received 11 September 2013; Revised 4 January 2014; Accepted 7 January 2014; Published 20 February 2014

Academic Editor: Shan Zhao

Copyright (C) 2014 Haitao Song et al. This is an open access article distributed under the Creative Commons Attribution License, which permits unrestricted use, distribution, and reproduction in any medium, provided the original work is properly cited.

A computer virus model with infection delay and recovery delay is considered. The sufficient conditions for the global stability of the virus infection equilibrium are established. We show that the time delay can destabilize the virus infection equilibrium and give rise to Hopf bifurcations and stable periodic orbits. By the normal form and center manifold theory, the direction of the Hopf bifurcation and stability of the bifurcating periodic orbits are determined. Numerical simulations are provided to support our theoretical conclusions.

\section{Introduction}

In recent years, the computer networks have become more and more popular, and people can find many useful things through computer networks. However, computer virus flows and spoils the correct operation of computer. As the computer networks become necessary tools in our daily life, computer virus becomes a major threat [1].

Cohen [2] found that there is high similarity between biological virus and computer virus. By the Kermack and McKendrick SIR epidemic model [3], the computer virus models were proposed in [4-7] which analyzed the spread of computer virus by epidemiological models. The model is as follows:

$$
\begin{gathered}
\frac{\mathrm{d} S(t)}{\mathrm{d} t}=b-\beta S(t) I(t)-\mu S(t), \\
\frac{\mathrm{d} I(t)}{\mathrm{d} t}=\beta S(t) I(t)-(\mu+\gamma) I(t), \\
\frac{\mathrm{d} R(t)}{\mathrm{d} t}=\gamma I(t)-\mu R(t),
\end{gathered}
$$

where $S(t), I(t)$, and $R(t)$ denote susceptible, infected, and recovered computers, respectively. Here we assume that all the computers connect to the network. $b$ is the rate at which external computers are connected to the network, $\gamma$ is the recovery rate of infected computers because of the antivirus ability of the network, $\beta$ is the infection rate, and $\mu$ is the death rate of the classes $S(t), I(t)$, and $R(t)$.

Dong et al. [8] considered the effect of immunization on susceptible state and exposed state by a delayed computer virus model. Zhang et al. [9] studied an impulse model for computer viruses and established the global dynamics of the model. Yang et al. [10] analyzed a computer virus model with graded cure rates and showed that the global dynamics are determined by the basic reproduction number. Then we can understand and control the computer virus propagation using the mathematical models.

Since there is a period of time from virus entering a host to active state [11], there exists an infection delay from infected to infectious computers. Similarly, there is a recovery delay from recovered to susceptible computers. Then the model is

$$
\begin{gathered}
\frac{\mathrm{d} S(t)}{\mathrm{d} t}=b-\beta S(t) I\left(t-\tau_{1}\right) e^{-\mu \tau_{1}}+\nu R\left(t-\tau_{2}\right)-\mu S(t), \\
\frac{\mathrm{d} I(t)}{\mathrm{d} t}=\beta S(t) I\left(t-\tau_{1}\right) e^{-\mu \tau_{1}}-(\mu+\gamma) I(t), \\
\frac{\mathrm{d} R(t)}{\mathrm{d} t}=\gamma I(t)-\nu R\left(t-\tau_{2}\right)-\mu R(t),
\end{gathered}
$$


where $0<e^{-\mu \tau_{1}} \leq 1$ is the survival probability of the infected computers, $\tau_{1}$ is the infection delay, and $v$ is the rate at which one recovered computer reverts to the susceptible one. $R\left(t-\tau_{2}\right)$ denotes that a recovered computer moves into the susceptible class after time $\tau_{2}$.

Ren et al. [12] obtained that the virus-free equilibrium is globally asymptotically stable when $R_{0}<1$. When $R_{0}>1$, we make a lot of improvement in the results in [12]. Compared to [12], we show that the virus infection equilibrium is always locally asymptotically stable for $\tau_{1}>0$ and $\tau_{2}=0$, and Hopf bifurcation does not exist. For $\tau_{1}>0$ and $\tau_{2}>0$, Ren et al. [12] constructed a Lyapunov function by linearized equations of $E^{*}$ and obtained the sufficient conditions for global stability of the virus infection equilibrium. Obviously, this is inappropriate. Then we construct a suitable Lyapunov function and obtain the sufficient conditions of global stability for the virus infection equilibrium when $\tau_{1}>0$ and $\tau_{2}>0$. Furthermore, for $\tau_{1}=0$ and $\tau_{2}>0$, we study the direction of the Hopf bifurcation and stability of the bifurcating periodic orbits by the normal form and center manifold theory, and numerical simulations are given to support the theoretical conclusions.

Then we can control the computer virus propagation using the epidemiological threshold value. There are many research works about epidemiological models; see [13-23] and the references therein. For example, $\mathrm{Ma}$ et al. [24] analyzed the global stability of a SIR epidemic model with a time delay. Wang and Zhao [25] obtained the basic reproduction number of reaction-diffusion epidemic models with compartmental structure and considered the influence of spatial heterogeneity and population mobility on disease transmission by a spatial model. The methods used here are Lyapunov functions, normal form, and center manifold theory. There are many research papers using the method of constructing Lyapunov functions [26-33]. In addition, we would like to refer to some excellent articles about stability and bifurcation; see [34-44].

The paper is organized as follows. In Section 2, the existence of equilibria is discussed and characteristic equation is given. In Section 3, we establish the local stability of the virus infection equilibrium $E^{*}$ for $\tau_{1}>0$ and $\tau_{2}=0$. In Section 4 , for $\tau_{1}=0$ and $\tau_{2}>0$, we consider the local stability of $E^{*}$ and existence of local Hopf bifurcation, and the direction of Hopf bifurcation and stability of the bifurcating periodic solutions are considered. In Section 5, for $\tau_{1}>0$ and $\tau_{2}>0$, global stability of the virus infection equilibrium is obtained. Finally, we finish the paper with conclusions.

\section{Preliminaries}

The initial conditions are

$$
\begin{array}{r}
S(\theta)=\varphi_{1}(\theta) \geq 0, \\
I(\theta)=\varphi_{2}(\theta) \geq 0, \\
R(\theta)=\varphi_{3}(\theta) \geq 0, \\
\theta \in[-\tau, 0],
\end{array}
$$

where $\tau=\max \left\{\tau_{1}, \tau_{2}\right\}$ and $\varphi=\left(\varphi_{1}, \varphi_{2}, \varphi_{3}\right) \in \mathscr{C}\left([-\tau, 0], \mathbb{R}^{3}\right)$ with $\varphi_{i}(0)>0(i=1,2,3)$. We denote by $X=\mathscr{C}\left([-\tau, 0], \mathbb{R}^{3}\right)$ the Banach space of continuous functions mapping the internal $[-\tau, 0]$ into $\mathbb{R}^{3}$ equipped with the sup-norm $\|\varphi\|=$ $\sup _{\theta \in[-\tau, 0]}|\varphi(\theta)|$ for $\varphi \in X$.

From the standard theory of functional differential equation [45], for all $\varphi \in X$, there is a unique solution $(S(t, \varphi), I(t, \varphi), R(t, \varphi))$ with $\varphi_{i}(0)>0(i=1,2,3)$ of the system (2). By the method of [13], under the initial conditions (3), all solutions of system (2) are positive and ultimately bounded in $\mathbb{R} \times X \times X$. The feasible region of system (2) is

$$
\Gamma=\left\{(S, I, R): S \geq 0, I \geq 0, R \geq 0, S+I+R \leq \frac{b}{\mu}\right\}
$$

which is the positively invariant set with respect to system (2). System (2) always has a virus-free equilibrium $E_{0}=$ $\left(S_{0}, 0,0\right)$ with $S_{0}=b / \mu$. In addition, system (2) has a virus infection equilibrium $E^{*}=\left(S^{*}, I^{*}, R^{*}\right)$. The basic reproduction number is defined [12] as

$$
R_{0}=\frac{\beta e^{-\mu \tau_{1}} S_{0}}{\mu+\gamma} .
$$

The virus-free equilibrium $E_{0}$ always exists. The coordinates of the virus infection equilibrium $E^{*}=\left(S^{*}, I^{*}, R^{*}\right)$ are given by

$$
\begin{gathered}
S^{*}=\frac{b}{\mu R_{0}}, \quad I^{*}=\frac{b\left(R_{0}-1\right)(\mu+\nu)}{R_{0}[(\mu+\gamma)(\mu+\nu)-\gamma \nu]}, \\
R^{*}=\frac{\gamma b\left(R_{0}-1\right)}{R_{0}[(\mu+\gamma)(\mu+\nu)-\gamma \nu]} .
\end{gathered}
$$

Thus, $E^{*}$ exists in the interior of $\Gamma$ if and only if $R_{0}>1$.

Let $x(t)=S(t)-S^{*}, y(t)=I(t)-I^{*}$, and $z(t)=R(t)-R^{*}$. Then system (2) becomes

$$
\begin{gathered}
\dot{x}(t)=-\mu x(t)-\beta I^{*} e^{-\mu \tau_{1}} x(t)-\beta S^{*} e^{-\mu \tau_{1}} y\left(t-\tau_{1}\right) \\
+\nu z\left(t-\tau_{2}\right)-\beta e^{-\mu \tau_{1}} x(t) y\left(t-\tau_{1}\right), \\
\dot{y}(t)=\beta I^{*} e^{-\mu \tau_{1}} x(t)-(\mu+\gamma) y(t)+\beta S^{*} e^{-\mu \tau_{1}} y\left(t-\tau_{1}\right) \\
+\beta e^{-\mu \tau_{1}} x(t) y\left(t-\tau_{1}\right), \\
\dot{z}(t)=\gamma y(t)-\mu z(t)-\nu z\left(t-\tau_{2}\right) .
\end{gathered}
$$

The characteristic equation of system $(7)$ at $(0,0,0)$ is

$$
\begin{gathered}
(\lambda+\mu)\left[\lambda^{2}+\lambda\left(2 \mu+\gamma+\beta I^{*} e^{-\mu \tau_{1}}\right)+\mu^{2}+\beta I^{*} \gamma e^{-\mu \tau_{1}}\right. \\
\left.+\mu \beta I^{*} e^{-\mu \tau_{1}}+\gamma \mu\right]+(\lambda+\mu) \\
\times\left[e^{-\lambda \tau_{1}}\left(-\beta S^{*} e^{-\mu \tau_{1}} \lambda-\beta S^{*} e^{-\mu \tau_{1}} \mu\right)+e^{-\lambda \tau_{2}}\right. \\
\left.\times\left(\nu \lambda+\mu \nu+\beta I^{*} \nu e^{-\mu \tau_{1}}+\gamma \nu\right)\right] \\
+(\lambda+\mu)\left[e^{-\lambda\left(\tau_{1}+\tau_{2}\right)}\left(-\beta S^{*} \nu e^{-\mu \tau_{1}}\right)\right]=0 .
\end{gathered}
$$




\section{Local Stability of $E^{*}$ for $\tau_{1}>0$ and $\tau_{2}=0$}

Consider $\tau_{1}>0$ and $\tau_{2}=0$; then (8) becomes

$$
(\lambda+\mu)\left[\lambda^{2}+p_{1} \lambda+p_{0}+e^{-\lambda \tau_{1}}\left(q_{1} \lambda+q_{0}\right)\right]=0,
$$

where

$$
\begin{aligned}
& p_{1}=(\mu+\gamma)+(\mu+\nu)+\beta I^{*} e^{-\mu \tau_{1}} \\
& p_{0}=(\mu+\gamma)(\mu+\nu)+(\mu+\gamma+\nu) \beta I^{*} e^{-\mu \tau_{1}}, \\
& q_{1}=-\beta S^{*} e^{-\mu \tau_{1}} \\
& q_{0}=-(\mu+\nu) \beta S^{*} e^{-\mu \tau_{1}} .
\end{aligned}
$$

Since $\lambda=-\mu$ is a negative real root of (9), all other roots of (9) are given by

$$
\lambda^{2}+p_{1} \lambda+p_{0}+e^{-\lambda \tau_{1}}\left(q_{1} \lambda+q_{0}\right)=0 .
$$

If $\lambda=i \omega\left(\tau_{1}\right)(\omega>0)$ is the purely imaginary root of (11), then substituting $\lambda=i \omega$ into (11) and separating the real and imaginary parts yield

$$
\begin{gathered}
p_{1} \omega=q_{0} \sin \omega \tau_{1}-q_{1} \omega \cos \omega \tau_{1}, \\
\omega^{2}-p_{0}=q_{1} \omega \sin \omega \tau_{1}+q_{0} \cos \omega \tau_{1} .
\end{gathered}
$$

Squaring and adding the two equations lead to

$$
\omega^{4}+\left(p_{1}^{2}-2 p_{0}-q_{1}^{2}\right) \omega^{2}+p_{0}^{2}-q_{0}^{2}=0
$$

Note that

$$
\begin{gathered}
p_{0}^{2}-q_{0}^{2}=\left[2(\mu+\gamma)(\mu+\nu)+(\mu+\gamma+\nu) \beta I^{*} e^{-\mu \tau_{1}}\right] \\
\times(\mu+\gamma+\nu) \beta I^{*} e^{-\mu \tau_{1}}>0, \\
p_{1}^{2}-2 p_{0}-q_{1}^{2}=(\mu+\nu)^{2}+\left(\beta I^{*} e^{-\mu \tau_{1}}\right)^{2}+2 \mu \beta I^{*} e^{-\mu \tau_{1}}>0 .
\end{gathered}
$$

Therefore, the roots of (13) do not exist. Thus, the virus infection equilibrium $E^{*}$ of system (2) is locally asymptotically stable for $\tau_{1}>0$ and $\tau_{2}=0$. Then we obtain the following result.

Theorem 1. Consider system (2). If $R_{0}>1$, then the virus infection equilibrium $E^{*}$ is locally asymptotically stable for $\tau_{1}>$ 0 and $\tau_{2}=0$.

Remark 2. For $\tau_{1}>0$ and $\tau_{2}=0$, Ren et al. [12] showed that the time delay $\tau_{1}$ can destabilize the infected equilibrium $E^{*}$ leading to Hopf bifurcations. However, we show that the virus infection equilibrium $E^{*}$ is always locally asymptotically stable for $\tau_{1}>0$ and $\tau_{2}=0$, and Hopf bifurcation does not exist.

We introduce a set of parameter values: $b=0.2, \beta=0.3$, $\mu=0.1, \gamma=0.2, \nu=0.5$, and $\tau_{2}=0$. Consequently, (a) $R_{0}=1.8097>1$ and $E^{*}=(1.1052,0.6711,0.2237)$;

(b) $R_{0}=1.6375>1$ and $E^{*}=(1.2214,0.5840,0.1947)$;

(c) $R_{0}=1.4816>1$ and $E^{*}=(1.3499,0.4876,0.1625)$;

(d) $R_{0}=1.2131>1$ and $E^{*}=(1.6487,0.2635,0.0878)$.

Figure 1 shows that the infection equilibrium $E^{*}$ is locally asymptotically stable.

\section{Hopf Bifurcation for $\tau_{1}=0$ and $\tau_{2}>0$}

Consider $\tau_{1}=0$ and $\tau_{2}>0$; then (8) becomes

$$
(\lambda+\mu)\left[\lambda^{2}+a_{1} \lambda+a_{2}+e^{-\lambda \tau_{2}}\left(b_{1} \lambda+b_{2}\right)\right]=0,
$$

where

$$
\begin{gathered}
a_{1}=2 \mu+\gamma+\beta I^{*}-\beta S^{*}, \\
a_{2}=\mu\left(\mu+\gamma+\beta I^{*}-\beta S^{*}\right)+\beta I^{*} \gamma, \\
b_{1}=\nu, \\
b_{2}=\nu\left(\mu+\gamma+\beta I^{*}-\beta S^{*}\right) .
\end{gathered}
$$

Obviously, $\lambda=-\mu$ is always a negative real root of (15). Then we consider

$$
\lambda^{2}+a_{1} \lambda+a_{2}+e^{-\lambda \tau_{2}}\left(b_{1} \lambda+b_{2}\right)=0 .
$$

If $\lambda=i \omega\left(\tau_{2}\right)(\omega>0)$ is the purely imaginary root of (17), then substituting $\lambda=i \omega$ into (17) and separating the real and imaginary parts give

$$
\begin{gathered}
\omega^{2}-a_{2}=b_{2} \cos \omega \tau_{2}+b_{1} \omega \sin \omega \tau_{2}, \\
a_{1} \omega=b_{2} \sin \omega \tau_{2}-b_{1} \omega \cos \omega \tau_{2}
\end{gathered}
$$

Squaring and adding the two equations lead to

$$
\omega^{4}+\left(a_{1}^{2}-2 a_{2}-b_{1}^{2}\right) \omega^{2}+a_{2}^{2}-b_{2}^{2}=0 .
$$

Denote

$$
f(u)=u^{2}+\left(a_{1}^{2}-2 a_{2}-b_{1}^{2}\right) u+a_{2}^{2}-b_{2}^{2}=0 .
$$

Let

$$
\Delta=\left(a_{1}^{2}-2 a_{2}-b_{1}^{2}\right)^{2}-4\left(a_{2}^{2}-b_{2}^{2}\right) .
$$

Hence

(1) if $\Delta<0$, then (20) has no positive real roots;

(2) if $a_{2}^{2}-b_{2}^{2}<0$, since $\lim _{u \rightarrow \infty} f(u)=\infty$, then (20) has at least one positive real root;

(3) if $\Delta \geq 0$, then (20) has two real roots:

$$
\begin{aligned}
& u_{1}=\frac{-\left(a_{1}^{2}-2 a_{2}-b_{1}^{2}\right)+\sqrt{\Delta}}{2}, \\
& u_{2}=\frac{-\left(a_{1}^{2}-2 a_{2}-b_{1}^{2}\right)-\sqrt{\Delta}}{2} ;
\end{aligned}
$$

if $u_{1}>0$, then (20) has a positive real root. If $u_{1}<0$, then all roots of (20) are negative.

Let $u_{i}(i=1,2)$ be the positive real roots such that $f(u)=$ 0 and $\omega_{i}=\sqrt{u_{i}}(i=1,2)$. Denote

$$
\tau_{i}^{k}=\frac{\theta+2 k \pi}{\omega_{i}}, \quad i=1,2, k=1,2, \ldots, n,
$$




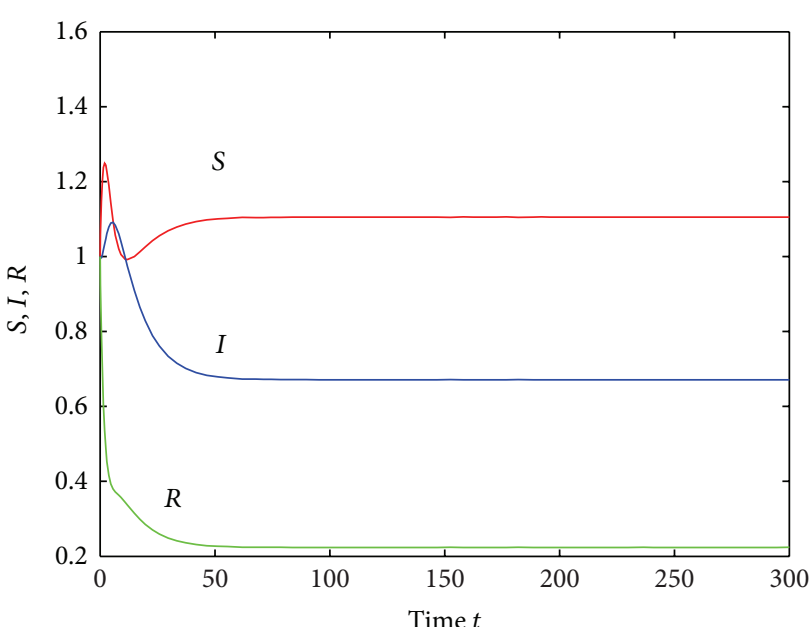

(a)

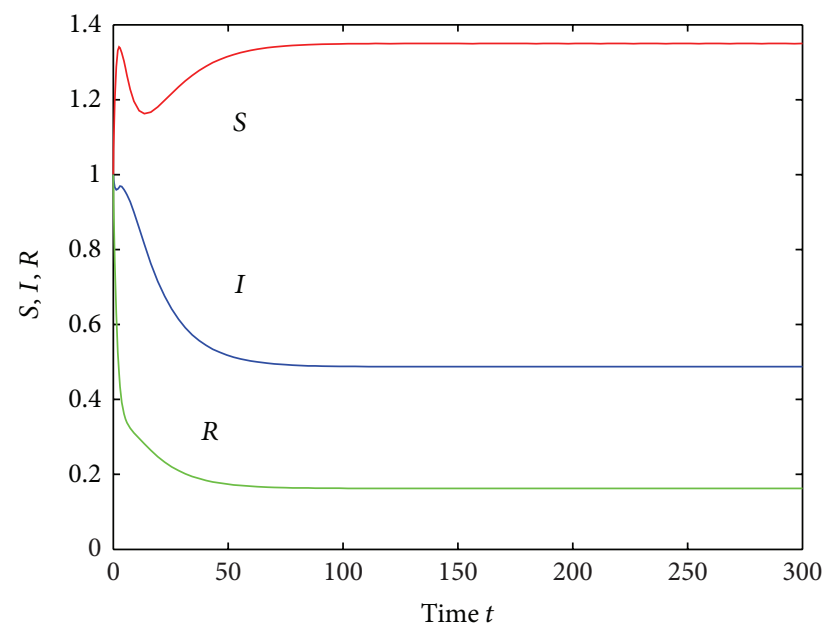

(c)

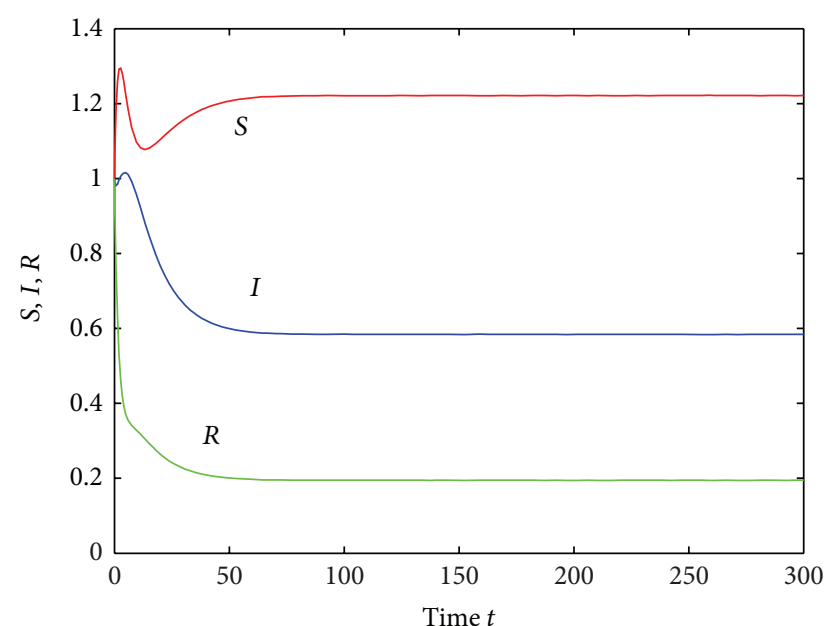

(b)

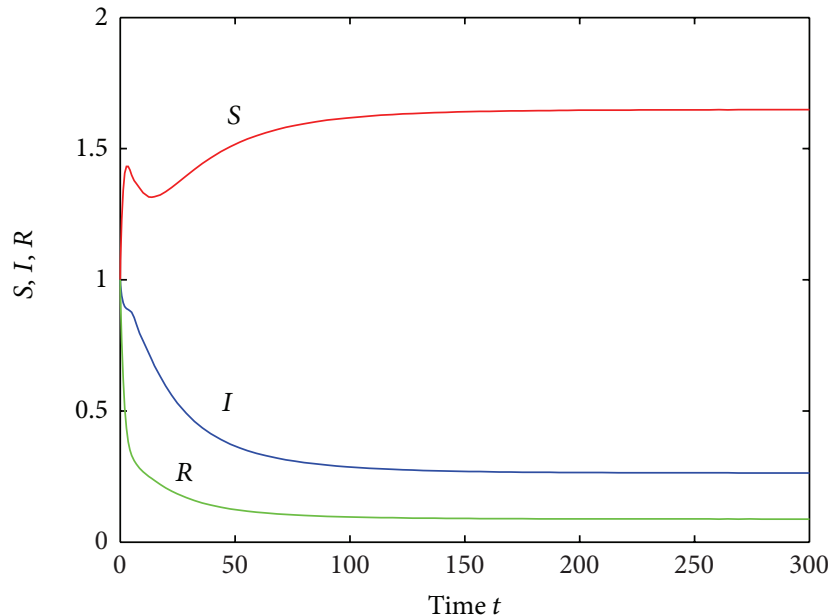

(d)

FIGURE 1: In the case of $\tau_{1}>0$ and $\tau_{2}=0$, numerical simulations for system (2). The initial values are $S(0)=1, I(0)=1$, and $R(0)=1$. (a) $\tau_{1}=1$; (b) $\tau_{1}=2$; (c) $\tau_{1}=3$; (d) $\tau_{1}=5$. Other parameter values are given in the text, and the infection equilibrium is asymptotically stable.

where $\theta \in[0,2 \pi]$ is determined by

$$
\begin{gathered}
\cos \theta=\frac{\left(b_{2}-a_{1} b_{1}\right) \omega_{i}^{2}-a_{2} b_{2}}{b_{1}^{2} \omega_{i}^{2}+b_{2}^{2}}, \\
\sin \theta=\frac{b_{1} \omega_{i}^{3}+\left(a_{1} b_{2}-a_{2} b_{1}\right) \omega_{i}}{b_{1}^{2} \omega_{i}^{2}+b_{2}^{2}}, \quad i=1,2 .
\end{gathered}
$$

Define

$$
\tau_{0}=\min _{1 \leq i \leq 2}\left\{\tau_{i}^{k} \mid f\left(u_{i}\right)=0\right\}, \quad \omega_{0}=\omega_{k}, \quad k=1,2, \ldots, n .
$$

Therefore, $\tau_{0}$ is the first value of $\tau$ when a pair of characteristic roots cross the imaginary axis at $\pm i \omega_{0}$.
Let $\lambda\left(\tau_{2}\right)=\alpha\left(\tau_{2}\right)+i \omega\left(\tau_{2}\right)$ be the root of (15) satisfying $\alpha\left(\tau_{i}^{k}\right)=0$ and $\omega\left(\tau_{i}^{k}\right)=\omega_{0}$. Differentiating (15) with respect to $\tau_{2}$ leads to

$$
\left\{\frac{\mathrm{d} \lambda}{\mathrm{d} \tau_{2}}\right\}^{-1}=\frac{2 \lambda+a_{1}}{\left(b_{1} \lambda+b_{2}\right) e^{-\lambda \tau_{2}}}+\frac{b_{1}}{\left(b_{1} \lambda+b_{2}\right) \lambda}-\frac{\tau_{2}}{\lambda} .
$$

From (18), we have

$$
\left\{\frac{\left(\mathrm{dRe} \lambda\left(\tau_{2}\right)\right)}{\mathrm{d} \tau_{2}}\right\}_{\tau_{2}=\tau_{0}}^{-1}=\frac{2 \omega_{0}^{2}+a_{1}^{2}-2 a_{2}-b_{1}^{2}}{b_{1}^{2} \omega_{0}^{2}+b_{2}^{2}}=\frac{f^{\prime}\left(\omega_{0}^{2}\right)}{b_{1}^{2} \omega_{0}^{2}+b_{2}^{2}} .
$$

Then

$$
\operatorname{sign}\left\{\frac{\left(\operatorname{dRe} \lambda\left(\tau_{2}\right)\right)}{\mathrm{d} \tau_{2}}\right\}_{\tau_{2}=\tau_{0}}^{-1}=\operatorname{sign} f^{\prime}\left(\omega_{0}^{2}\right) .
$$

Thus, we obtain the following the result. 
Theorem 3. Consider system (2). In the case of $\tau_{1}=0$ and $\tau_{2}>0$,

(i) assume that one of the following two conditions holds:

(a) $\Delta<0$.

(b) $\Delta \geq 0$ and $u_{1}<0$.

Then the virus infection equilibrium $E^{*}$ is locally asymptotically stable for $\tau_{2}>0$;

(ii) in either case,

(a) $a_{2}^{2}-b_{2}^{2}<0$, or

(b) $\Delta \geq 0$ and $u_{1}>0$,

the virus infection equilibrium $E^{*}$ is locally asymptotically stable for $\tau_{2} \in\left[0, \tau_{0}\right)$. Furthermore, if $f^{\prime}\left(\omega_{0}^{2}\right) \neq 0$, then system (2) undergoes a Hopf bifurcation at the virus infection equilibrium $E^{*}$ when $\tau_{2}=\tau_{0}$.

From the above discussions, the sufficient conditions for the existence of Hopf bifurcation were given for $\tau_{1}=0$ and $\tau_{2}>0$. Thus, under the conditions of Theorem 3 , we will study the direction of the Hopf bifurcation and the stability of the bifurcating periodic orbits by normal form and center manifold theory (see, e.g., [46]).

Fix $\tau_{1}=0$ and let $\tau_{2}=\tau_{0}+\mu_{0}$, then $\mu_{0}=0$ is the Hopf bifurcation value, and system (47) is transformed into an FDE in $C=C\left([-1,0], \mathbb{R}^{3}\right)$ as

$$
\dot{x}(t)=L_{\mu}\left(x_{t}\right)+f\left(\mu_{0}, x_{t}\right),
$$

where $x_{t}(\theta)=x(t+\theta)$. Denote $L_{\mu}: C \rightarrow \mathbb{R}^{3}$ as

$$
L_{\mu} \varphi=\left(\tau_{0}+\mu_{0}\right) A_{1} \varphi(0)+\left(\tau_{0}+\mu_{0}\right) A_{2} \varphi(-1),
$$

where

$$
\begin{gathered}
A_{1}=\left(\begin{array}{ccc}
-\mu-\beta I^{*} & -\beta S^{*} & 0 \\
\beta I^{*} & \beta S^{*}-(\mu+\gamma) & 0 \\
0 & \gamma & -\mu
\end{array}\right), \\
A_{2}=\left(\begin{array}{ccc}
0 & 0 & v \\
0 & 0 & 0 \\
0 & p & -v
\end{array}\right), \\
f\left(\mu_{0}, \varphi\right)=\left(\tau_{0}+\mu_{0}\right)\left(\begin{array}{c}
-\beta \varphi_{1}(0) \varphi_{2}(0) \\
\beta \varphi_{1}(0) \varphi_{2}(0) \\
0
\end{array}\right) .
\end{gathered}
$$

Define

$$
\eta\left(\theta, \mu_{0}\right)=\left(\tau_{0}+\mu_{0}\right) A_{1} \delta(\theta)-\left(\tau_{0}+\mu_{0}\right) A_{2} \delta(\theta+1),
$$

where

$$
\delta(\theta)= \begin{cases}0, & \theta \neq 0 \\ 1, & \theta=0 .\end{cases}
$$

Then we can denote $L_{\mu}$ as

$$
L_{\mu} \varphi=\int_{-1}^{0} \mathrm{~d} \eta\left(\theta, \mu_{0}\right) \varphi(\theta), \quad \forall \varphi \in C\left([-1,0], \mathbb{R}^{3}\right) .
$$

For $\varphi \in C^{1}\left([-1,0], \mathbb{R}^{3}\right)$, we define

$$
\begin{gathered}
A\left(\mu_{0}\right) \varphi= \begin{cases}\frac{\mathrm{d} \varphi(\theta)}{\mathrm{d} \theta}, & \theta \in[-1,0), \\
\int_{-1}^{0} \mathrm{~d} \eta\left(s, \mu_{0}\right) \varphi(s), & \theta=0,\end{cases} \\
R\left(\mu_{0}\right) \varphi= \begin{cases}0, & \theta \in[-1,0), \\
f\left(\mu_{0}, \varphi\right), & \theta=0 .\end{cases}
\end{gathered}
$$

Therefore, system (47) becomes

$$
\dot{x}_{t}=A\left(\mu_{0}\right) x_{t}+R\left(\mu_{0}\right) x_{t},
$$

where $x_{t}(\theta)=x(t+\theta)$ for $\theta \in[-1,0)$. For $\psi \in\left([0,1],\left(\mathbb{R}^{3}\right)^{*}\right)$, define

$$
A^{*} \psi(s)= \begin{cases}-\frac{\mathrm{d} \varphi(s)}{\mathrm{d} s}, & \theta \in[0,1), \\ \int_{-1}^{0} \mathrm{~d} \eta^{T}(t, 0) \psi(-t), & s=0 .\end{cases}
$$

and a bilinear form

$$
\begin{aligned}
\langle\psi(s), \varphi(\theta)\rangle= & \bar{\psi}(0) \varphi(0) \\
& -\int_{-1}^{0} \int_{\xi=0}^{\theta} \bar{\psi}(\xi-\theta) \mathrm{d} \eta(\theta) \varphi(\xi) \mathrm{d} \xi
\end{aligned}
$$

where $\eta(\theta)=\eta(\theta, 0)$. Hence, $A(0)$ and $A^{*}$ are adjoint operators. By the above discussions, we know that $\pm i \omega_{0}$ are eigenvalues of $A(0)$ and so they are also eigenvalues of $A^{*}$. It can be easily proved that $q(\theta)=\left(1, q_{2}, q_{3}\right)^{T} e^{i \omega_{0} \tau_{0} \theta}, \theta \in[-1,0)$ is an eigenvector of $A(0)$ associated with the eigenvalue $i \omega_{0} \tau_{0}$, and $q^{*}(s)=D\left(1, q_{2}^{*}, q_{3}^{*}\right) e^{i \omega_{0} s}, s \in(0,1]$ is an eigenvector of $A^{*}$ associated with the eigenvalue $-i \omega_{0} \tau_{0}$. Denote

$$
\begin{gathered}
\left(1, q_{2}, q_{3}\right)^{T}=\left(1, \frac{\beta I^{*}}{i \omega_{0}-\beta S^{*}+\mu+\gamma},\right. \\
\left.\frac{\gamma \beta I^{*}}{\left(i \omega_{0}-\beta S^{*}+\mu+\gamma\right)\left(i \omega_{0}+\mu+e^{-i \omega_{0} \tau_{0}}\right)}\right)^{T}, \\
\left(1, q_{2}^{*}, q_{3}^{*}\right)=\left(1, \frac{\beta I^{*}}{-i \omega_{0}+\mu+\beta I^{*}}, \frac{\nu e^{i \omega_{0} \tau_{0}}}{-i \omega_{0}+\mu+\nu e^{i \omega_{0} \tau_{0}}}\right), \\
D=\left(1+\bar{q}_{2} q_{2}^{*}+\bar{q}_{3} q_{3}^{*}+v \bar{q}_{3}\left(1-q_{3}^{*}\right) \tau_{0} e^{i \omega_{0} \tau_{0}}\right)^{-1},
\end{gathered}
$$

where $\left\langle q^{*}(s), q(\theta)\right\rangle=1,\left\langle q^{*}(s), \bar{q}(\theta)\right\rangle=0$. 
Following the procedure in [46], we obtain the following coefficients:

$$
\begin{gathered}
g_{20}=2 \tau_{0} \bar{D} \beta q_{2}\left(\bar{q}_{2}^{*}-1\right), \\
g_{11}=\tau_{0} \bar{D} \beta\left(q_{2}+\bar{q}_{2}\right)\left(\bar{q}_{2}^{*}-1\right), \\
g_{02}=2 \tau_{0} \bar{D} \beta \bar{q}_{2}\left(\bar{q}_{2}^{*}-1\right), \\
g_{21}=2 \tau_{0} \bar{D} \beta\left(\bar{q}_{2}^{*}-1\right) \\
\times\left[\frac{1}{2} W_{20}^{1}(0) \bar{q}_{2}+W_{11}^{1}(0) q_{2}+W_{11}^{2}(0)+\frac{1}{2} W_{20}^{2}(0)\right],
\end{gathered}
$$

where

$$
\begin{gathered}
W_{20}(\theta)=\frac{i g_{20}}{\omega_{0} \tau_{0}} q(0) e^{i \omega_{0} \tau_{0} \theta}+\frac{i \bar{g}_{02}}{3 \omega_{0} \tau_{0}} \bar{q}(0) e^{-i \omega_{0} \tau_{0} \theta} \\
+E_{1} e^{2 i \omega_{0} \tau_{0} \theta}, \\
W_{11}(\theta)=-\frac{i g_{11}}{\omega_{0} \tau_{0}} q(0) e^{i \omega_{0} \tau_{0} \theta}+\frac{i \bar{g}_{11}}{\omega_{0} \tau_{0}} \bar{q}(0) e^{-i \omega_{0} \tau_{0} \theta}+E_{2}, \\
E_{1}=2 G_{1}^{-1}\left(\begin{array}{c}
-\beta q_{2} \\
\beta q_{2} \\
0
\end{array}\right),
\end{gathered}
$$

where

$$
\begin{gathered}
G_{1} \\
=\left(\begin{array}{ccc}
2 i \omega_{0}+\mu+\beta I^{*} & \beta S^{*} & -\nu e^{-2 i \omega_{0} \tau_{0}} \\
-\beta I^{*} & 2 i \omega_{0}-\beta S^{*}+\mu+\gamma & 0 \\
0 & -\gamma & 2 i \omega_{0}+\mu+\nu e^{-2 i \omega_{0} \tau_{0}}
\end{array}\right), \\
E_{2}=G_{2}^{-1}\left(\begin{array}{c}
-\beta\left(q_{2}+\bar{q}_{2}\right) \\
\beta\left(q_{2}+\bar{q}_{2}\right) \\
0
\end{array}\right),
\end{gathered}
$$

where

$$
G_{2}=\left(\begin{array}{ccc}
\mu+\beta I^{*} & \beta S^{*} & -\nu \\
-\beta I^{*} & -\beta S^{*}+\mu+\gamma & 0 \\
0 & -\gamma & \mu+\nu
\end{array}\right) .
$$

Then $g_{21}$ can be expressed definitely.

From (27), (40), and (41), we have

$$
\begin{gathered}
C_{1}(0)=\frac{i}{2 \omega_{0}}\left(g_{11} g_{20}-2\left|g_{11}\right|^{2}-\frac{\left|g_{02}\right|^{2}}{3}\right)+\frac{g_{21}}{2}, \\
\mu_{2}=-\frac{\operatorname{Re}\left(C_{1}(0)\right)}{\operatorname{Re}\left(\lambda^{\prime}\left(\tau_{0}\right)\right)} \\
\beta_{2}=2 \operatorname{Re}\left(C_{1}(0)\right) \\
T_{2}=-\frac{\operatorname{Im}\left(C_{1}(0)\right)+\mu_{2} \operatorname{Im}\left(\lambda^{\prime}\left(\tau_{0}\right)\right)}{\omega_{0}} .
\end{gathered}
$$

Then we have the following result.
Theorem 4. Assume that the conditions of Theorem 3 (ii) hold. Then $\mu_{2}$ determines the direction of the Hopf bifurcation: if $\mu_{2}>0\left(\mu_{2}<0\right)$, then the Hopf bifurcation is forward (backward) and the bifurcating periodic orbits exist for $\tau_{1}=0$ and $\tau_{2}>\tau_{0}\left(\tau_{2}<\tau_{0}\right) ; \beta_{2}$ determines the stability of bifurcating periodic orbits: the bifurcating orbits are asymptotically stable (unstable) when $\beta_{2}<0\left(\beta_{2}>0\right)$; and $T_{2}$ determines the period of the bifurcating periodic orbits: the period increases (decreases) when $T_{2}>0\left(T_{2}<0\right)$.

We introduce a set of parameter values: $b=0.8, \beta=0.3$, $\mu=0.1, \gamma=0.2$, and $\nu=0.5$. Consequently, $R_{0}=8.0000>$ 1 and $E^{*}=(1.0000,5.2500,1.7500)$. We can obtain that the conditions of Theorem 3 hold. When $\tau_{1}=0$, by direct computation we get $\omega_{0}=0.4660, \tau_{0}=4.6665, C_{1}(0)=$ $-0.2217-0.0354 i, \beta_{2}=-0.4434<0$, and $\mu_{2}=0.6722>$ 0 . By Theorem 3 we know that the stability of the infected equilibrium $E^{*}$ varies when $\tau_{2}$ increases and the infected equilibrium $E^{*}$ is asymptotically stable for $\tau_{2} \in[0,4.6665)$. Figure 2 illustrates the results. By Theorem 4 we know that the direction of the Hopf bifurcation is forward at $\tau_{2}=\tau_{0}=$ 4.6665. And Figure 3 illustrates the bifurcating periodic orbits are asymptotically stable.

\section{Global Asymptotic Stability}

The following result shows the global stability of the virus-free equilibrium.

Proposition 5 (see [12]). Consider the following.

If $R_{0}<1$, then the virus-free equilibrium of system (2) is globally asymptotically stable for all $\tau_{1}>0$ and $\tau_{2}>0$.

Next we will prove the global stability of the virus infection equilibrium of system (2). Let $x(t)=S(t)-S^{*}$, $y(t)=I(t)-I^{*}$, and $z(t)=R(t)-R^{*}$. Then system (2) becomes

$$
\begin{gathered}
\dot{x}(t)=-\mu x(t)-\beta I^{*} e^{-\mu \tau_{1}} x(t) \\
-\beta\left(x(t)+S^{*}\right) e^{-\mu \tau_{1}} y\left(t-\tau_{1}\right)+\nu z\left(t-\tau_{2}\right), \\
\dot{y}(t)=\beta I^{*} e^{-\mu \tau_{1}} x(t)-(\mu+\gamma) y(t) \\
\quad+\beta\left(x(t)+S^{*}\right) e^{-\mu \tau_{1}} y\left(t-\tau_{1}\right), \\
\dot{z}(t)=\gamma y(t)-\mu z(t)-\nu z\left(t-\tau_{2}\right) .
\end{gathered}
$$

From system (2) with initial conditions (3), we know that $x(t)+S^{*}=S(t) \leq b / \mu(\forall t \geq 0)$. By the method of constructing Lyapunov function [47], we obtain the following result.

Theorem 6. Consider system (2) with initial conditions (3). Assume that $R_{0}>1$, if

$$
\left(H_{1}\right) \nu<\mu<\frac{1}{2} \gamma+w \nu+\nu, 2(w+1)(\mu+\gamma)>\gamma+w \nu+\frac{2 \beta b}{\mu},
$$

where

$$
w=\frac{\beta I^{*} e^{-\mu \tau_{1}}}{2 \mu+\gamma}
$$



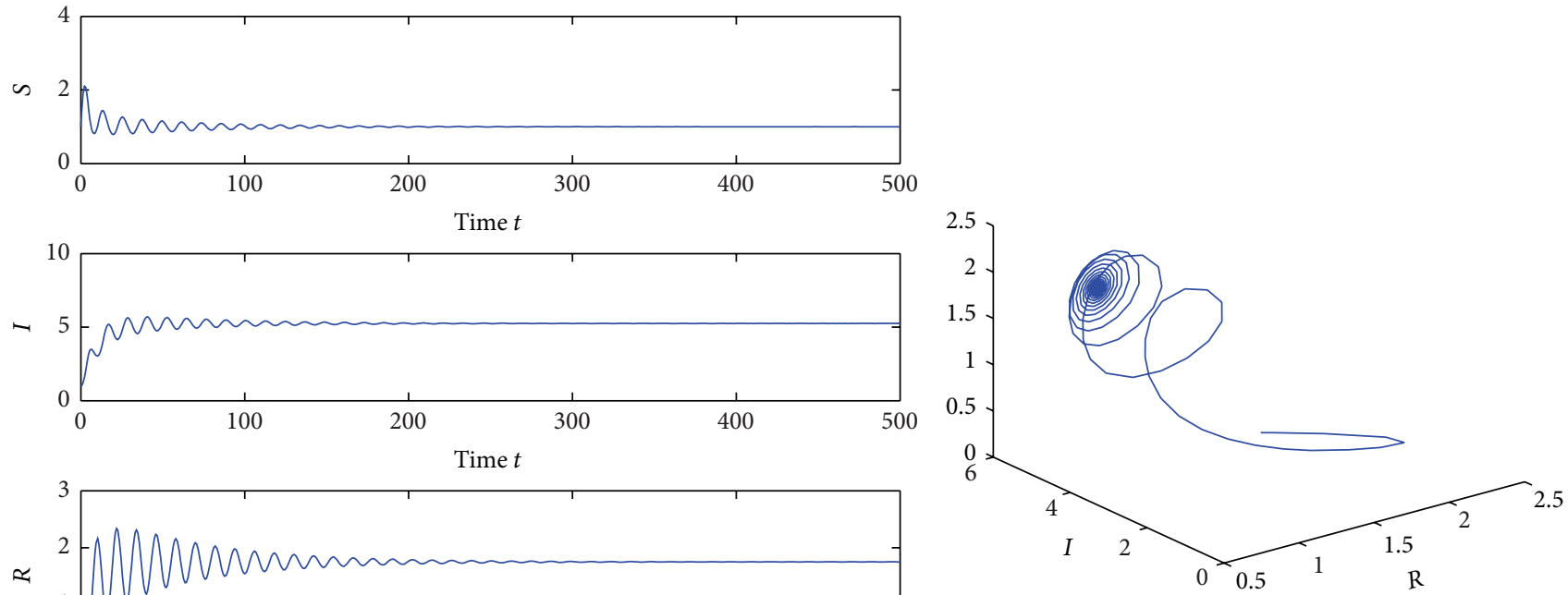

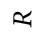

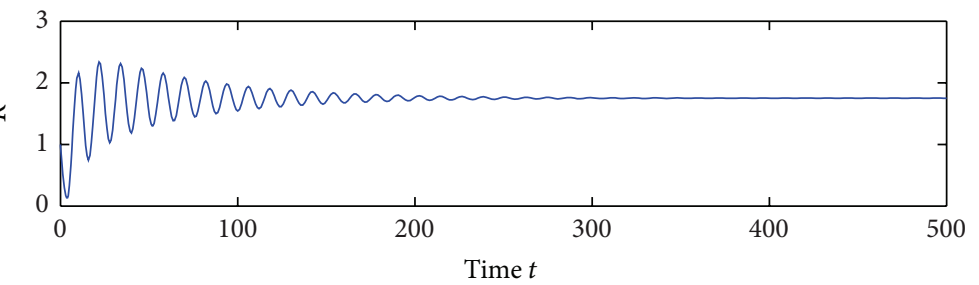

FIGURE 2: Behavior and phase portrait of system (2) with $\tau_{1}=0, \tau_{2}=4$. The virus infection equilibrium is asymptotically stable.
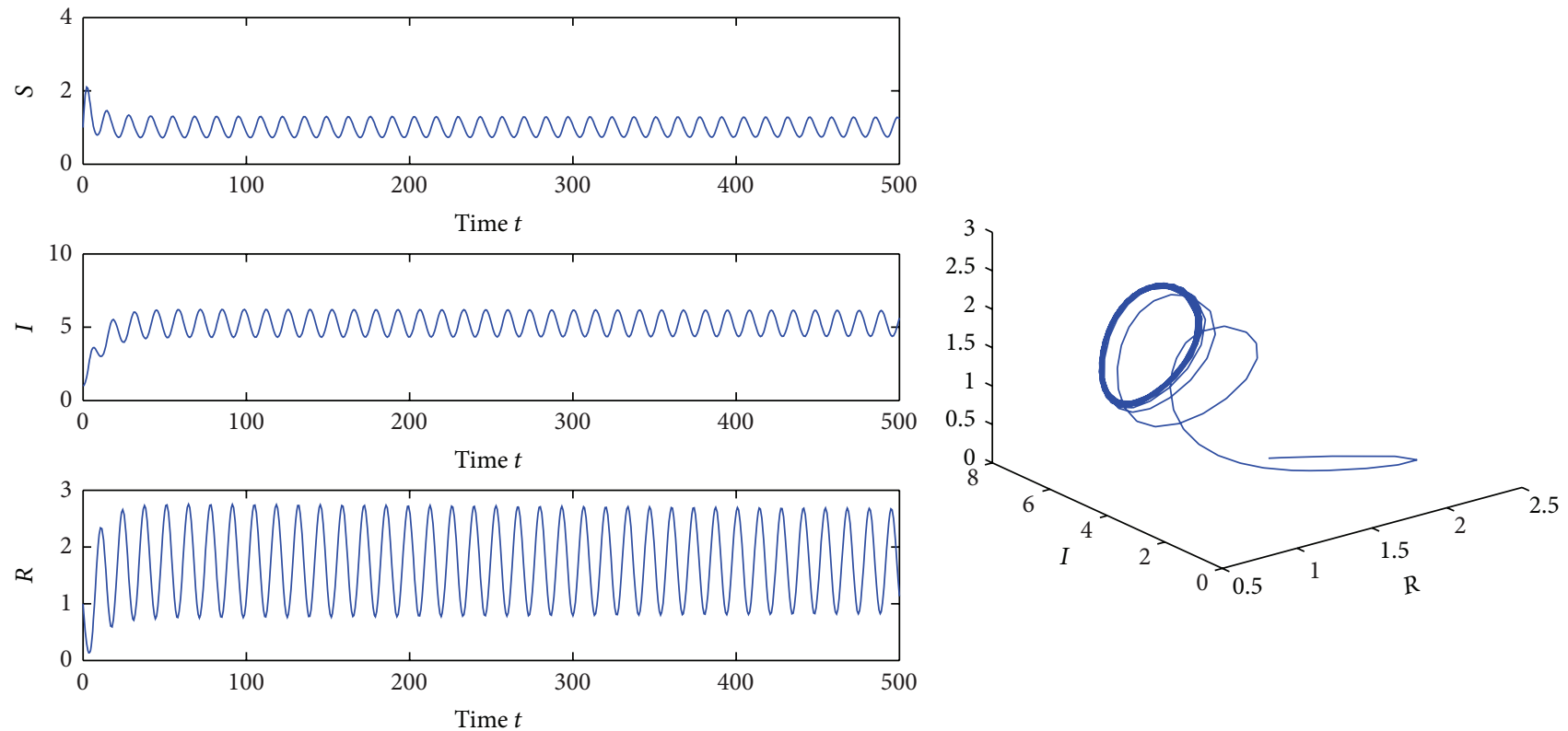

FIgURE 3: Behavior and phase portrait of system (2) with $\tau_{1}=0, \tau_{2}=5$. Hopf bifurcation occurs from the virus infection equilibrium.

holds, then the virus infection equilibrium $E^{*}$ is globally asymptotically stable for $\tau_{1}>0$ and $\tau_{2}>0$.

Proof. Define a Lyapunov function

$$
V_{1}(x, y, z)=\frac{1}{2} w(x+y)^{2}+\frac{1}{2}\left(y^{2}+z^{2}\right) \text {. }
$$

Differentiating $V_{1}(x, y, z)$ along the solution of system (47), we have

$$
\begin{aligned}
\left.\frac{\mathrm{d} V_{1}(x, y, z)}{\mathrm{d} t}\right|_{(5.1)} & =w(x+y)(\dot{x}+\dot{y})+y \dot{y}+z \dot{z} \\
& =w(x+y)\left[-\mu x+v z\left(t-\tau_{2}\right)-(\mu+\gamma) y\right]
\end{aligned}
$$

$$
\begin{aligned}
& +y\left[\beta\left(x(t)+S^{*}\right) y\left(t-\tau_{1}\right) e^{-\mu \tau_{1}}\right] \\
& +y\left[\beta I^{*} e^{-\mu \tau_{1}} x-(\mu+\gamma) y\right] \\
& +z\left[\gamma y-\mu z-\nu z\left(t-\tau_{2}\right)\right] \\
& =-w \mu x^{2}-w \mu x y+w \nu x z\left(t-\tau_{2}\right) \\
& +w \nu y z\left(t-\tau_{2}\right)-w(\mu+\gamma) x y \\
& -w(\mu+\gamma) y^{2}+\beta\left(x(t)+S^{*}\right) \\
& \times y y\left(t-\tau_{1}\right) e^{-\mu \tau_{1}}+\beta I^{*} e^{-\mu \tau_{1}} x y
\end{aligned}
$$




$$
\begin{aligned}
& -(\mu+\gamma) y^{2}+\gamma y z-\mu z^{2}-\nu z z\left(t-\tau_{2}\right) \\
= & -w \mu x^{2}-(w+1)(\mu+\gamma) y^{2}-\mu z^{2} \\
& +\left[\beta I^{*} e^{-\mu \tau_{1}}-w(2 \mu+\gamma)\right] x y+\gamma y z \\
& +w \nu x z\left(t-\tau_{2}\right)+w \nu y z\left(t-\tau_{2}\right)-\mu z^{2} \\
& +\beta\left(x(t)+S^{*}\right) y y\left(t-\tau_{1}\right) e^{-\mu \tau_{1}} .
\end{aligned}
$$

Since $x(t)+S^{*}=S(t) \leq b / \mu(\forall t \geq 0)$ and $\beta I^{*} e^{-\mu \tau_{1}}-w(2 \mu+$ $\gamma)=0$, and by Cauchy-Schwartz inequality we obtain

$$
\begin{aligned}
\left.\frac{\mathrm{d} V_{1}(x, y, z)}{\mathrm{d} t}\right|_{(5.1)} \leq & -w \mu x^{2}-(w+1)(\mu+\gamma) y^{2}-\mu z^{2} \\
& +\gamma y z+w \nu x z\left(t-\tau_{2}\right)+w \nu y z\left(t-\tau_{2}\right) \\
& -\mu z^{2}+\beta \frac{b}{\mu} y y\left(t-\tau_{1}\right) e^{-\mu \tau_{1}} \leq-w \mu x^{2} \\
& -(w+1)(\mu+\gamma) y^{2}-\mu z^{2}+\frac{1}{2} \gamma \\
& \times\left(y^{2}+z^{2}\right)+\frac{1}{2} w \nu\left[x^{2}+z^{2}\left(t-\tau_{2}\right)\right] \\
& +\frac{1}{2} w \nu\left[y^{2}+z^{2}\left(t-\tau_{2}\right)\right] \\
& +\frac{1}{2} \nu\left[z^{2}+z^{2}\left(t-\tau_{2}\right)\right] \\
& +\frac{b \beta e^{-\mu \tau_{1}}}{2 \mu}\left[y^{2}+y^{2}\left(t-\tau_{1}\right)\right]
\end{aligned}
$$

Define

$$
\begin{aligned}
V\left(x_{t}, y_{t}, z_{t}\right)= & V_{1}(x, y, z)+\frac{b \beta e^{-\mu \tau_{1}}}{2 \mu} \int_{-\tau_{1}}^{0} y^{2}(t+\theta) \mathrm{d} \theta \\
& +\left(w \nu+\frac{1}{2} \nu\right) \int_{-\tau_{2}}^{0} z^{2}(t+\theta) \mathrm{d} \theta
\end{aligned}
$$

Then

$$
\begin{aligned}
\left.\frac{\mathrm{d} V\left(x_{t}, y_{t}, z_{t}\right)}{\mathrm{d} t}\right|_{(5.1)} & \left.\frac{\mathrm{d} V_{1}(x, y, z)}{\mathrm{d} t}\right|_{(5.1)}+\frac{b \beta e^{-\mu \tau_{1}}}{2 \mu}\left[y^{2}-y^{2}\left(t-\tau_{1}\right)\right] \\
\leq & -\left(w \mu-\frac{1}{2} w \nu\right) x^{2}-\left(\mu-\frac{1}{2} \gamma-w \nu-\nu\right) z^{2} \\
& -\left[(w+1)(\mu+\gamma)-\frac{1}{2} \gamma-\frac{1}{2} w \nu-\frac{b \beta e^{-\mu \tau_{1}}}{2 \mu}\right] y^{2} .
\end{aligned}
$$

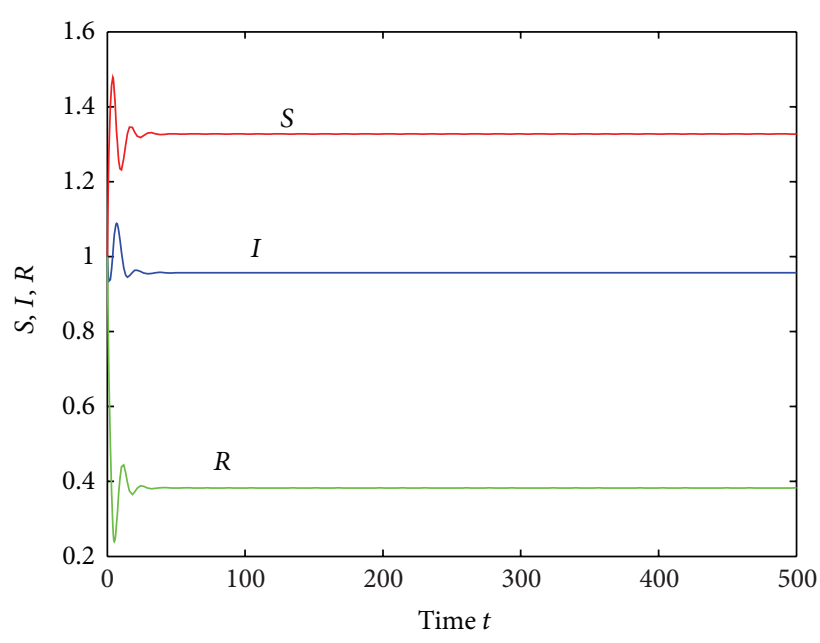

FIgURE 4: Numerical simulations of system (2) with parameter values: $b=0.8, \beta=0.4, \mu=0.3, \gamma=0.2$, and $\nu=0.2$, and $E^{*}$ is globally asymptotically stable.

Therefore, from $\left(H_{1}\right)$ we obtain

$$
\begin{gathered}
w \mu-\frac{1}{2} w \nu>0, \\
(w+1)(\mu+\gamma)-\frac{1}{2} \gamma-\frac{1}{2} w \nu-\frac{b \beta e^{-\mu \tau_{1}}}{2 \mu}>0, \\
\mu-\frac{1}{2} \gamma-w \nu-\nu>0 .
\end{gathered}
$$

Thus, $\left(H_{1}\right)$ ensures that $\mathrm{d} V\left(x_{t}, y_{t}, z_{t}\right) /\left.\mathrm{d} t\right|_{(5.1)} \leq 0$, and $\mathrm{d} V\left(x_{t}, y_{t}, z_{t}\right) /\left.\mathrm{d} t\right|_{(5.1)}=0$ if and only if $x=0, y=0$, and $z=0$. Then the only compact invariant set where $\left\{\mathrm{d} V\left(x_{t}, y_{t}, z_{t}\right) /\left.\mathrm{d} t\right|_{(5.1)}=0\right\}$ is the singleton $\{(0,0,0)\}$. By LaSalle's invariance principle [48], if $R_{0}>1$ and $\left(H_{1}\right)$ hold, then $E^{*}$ is globally asymptotically stable. The proof is completed.

Remark 7. For $\tau_{1}>0$ and $\tau_{2}>0$, Ren et al. constructed a Lyapunov function by linearized equations [12, equations (37)]. Clearly, this is unreasonable. Thus, we define an appropriate Lyapunov function and obtain the sufficient conditions of global stability for the virus infection equilibrium $E^{*}$.

We choose a set of parameter values: $b=0.8, \beta=0.4$, $\mu=0.3, \gamma=0.2$, and $\nu=0.2$. Correspondingly, $R_{0}=$ $2.1333>1$ and $E^{*}=(1.2500,1.0119,0.4048)$. It can be verified that the conditions of Theorem 6 are satisfied. For $\tau_{1}>0$ and $\tau_{2}>0$, we show that the infected equilibrium $E^{*}$ is globally asymptotically stable in Figure 4 . All of the numerical simulations are carried out by MATLAB.

\section{Conclusions}

We consider a computer virus model with infection delay and recovery delay. When $R_{0}<1$, the virus-free equilibrium of system (2) is globally asymptotically stable and the virus fades out from the network. When $R_{0}>1$, we obtain that the virus 
infection equilibrium is always locally asymptotically stable for $\tau_{1}>0$ and $\tau_{2}=0$; for $\tau_{1}=0$ and $\tau_{2}>0$, we establish that the time delay can destabilize the virus infection equilibrium and give rise to Hopf bifurcations and stable periodic orbits. For $\tau_{1}>0$ and $\tau_{2}>0$, we construct an appropriate Lyapunov function and get that the infection equilibrium is globally asymptotically stable under the sufficient conditions, and the virus finally persists at a constant endemic equilibrium level.

Our results show that we can take measures to make the basic reproduction number $R_{0}$ to be less than one leading to the extinction of computer virus. We must take some effective measures (such as the installation of antivirus software) to decrease the infection rate $\beta$ and increase recovery rate $\gamma$ of the infected computers.

However, our results should be viewed carefully because the model used in this paper is simplified and possibly does not explain all relevant dynamics of computer virus. Then we need more realistic computer virus models to study the real network.

\section{Acknowledgments}

The authors are grateful to the editor and the anonymous reviewers for their valuable comments and suggestions which greatly improved the paper. This research is supported by the National Natural Science Foundation of China (no. 11371112) and in part by the National Natural Science Foundation of China (no. 11031002) and by the Heilongjiang Provincial Natural Science Foundation (no. A201208).

\section{Conflict of Interests}

The authors declared that they have no conflict of interests to this work.

\section{References}

[1] P. J. Denning, Computers under Attack, Addison-Wesley, Reading, Mass, USA, 1990.

[2] F. Cohen, "Computer viruses. Theory and experiments," Computers and Security, vol. 6, no. 1, pp. 22-35, 1987.

[3] W. O. Kermack and A. G. McKendrick, "Contributions of mathematical theory to epidemics," Proceedings of the Royal Society A, vol. 141, pp. 94-122, 1933.

[4] J. C. Wierman and D. J. Marchette, "Modeling computer virus prevalence with a susceptible-infected-susceptible model with reintroduction," Computational Statistics \& Data Analysis, vol. 45, no. 1, pp. 3-23, 2004.

[5] J. R. C. Piqueira and V. O. Araujo, "A modified epidemiological model for computer viruses," Applied Mathematics and Computation, vol. 213, no. 2, pp. 355-360, 2009.

[6] B. K. Mishra and D. Saini, "Mathematical models on computer viruses," Applied Mathematics and Computation, vol. 187, no. 2, pp. 929-936, 2007.

[7] M. J. Keeling and K. T. D. Eames, "Networks and epidemic models," Journal of the Royal Society Interface, vol. 2, no. 4, pp. 295-307, 2005.
[8] T. Dong, X. Liao, and H. Li, "Stability and Hopf bifurcation in a computer virus model with multistate antivirus," Abstract and Applied Analysis, vol. 2012, Article ID 841987, 16 pages, 2012.

[9] C. Zhang, Y. Zhao, and Y. Wu, "An impulse model for computer viruses," Discrete Dynamics in Nature and Society, vol. 2012, Article ID 260962, 13 pages, 2012.

[10] L.-X. Yang, X. Yang, Q. Zhu, and L. Wen, "A computer virus model with graded cure rates," Nonlinear Analysis: Real World Applications, vol. 14, no. 1, pp. 414-422, 2013.

[11] Y. B. Kafai, "Understanding virtual epidemics: Children's folk conceptions of a computer virus," Journal of Science Education and Technology, vol. 17, no. 6, pp. 523-529, 2008.

[12] J. Ren, X. Yang, L.-X. Yang, Y. Xu, and F. Yang, "A delayed computer virus propagation model and its dynamics," Chaos, Solitons \& Fractals, vol. 45, no. 1, pp. 74-79, 2012.

[13] H. R. Thieme and P. van den Driessche, "Global stability in cyclic epidemic models with disease fatalities," in Differential Equations with Applications to Biology (Halifax, NS, 1997), vol. 21 of Fields Inst. Commun., pp. 459-472, Amer. Math. Soc., Providence, RI, USA, 1999.

[14] G. Li and Z. Jin, "Global stability of a SEIR epidemic model with infectious force in latent, infected and immune period," Chaos, Solitons \& Fractals, vol. 25, no. 5, pp. 1177-1184, 2005.

[15] H. Laarabi, E. H. Labriji, M. Rachik, and A. Kaddar, "Optimal control of an epidemic model with a saturated incidence rate," Nonlinear Analysis: Modelling and Control, vol. 17, no. 4, pp. 448-459, 2012.

[16] K. Cooke, P. van den Driessche, and X. Zou, "Interaction of maturation delay and nonlinear birth in population and epidemic models," Journal of Mathematical Biology, vol. 39, no. 4, pp. 332-352, 1999.

[17] S. Ruan and W. Wang, "Dynamical behavior of an epidemic model with a nonlinear incidence rate," Journal of Differential Equations, vol. 188, no. 1, pp. 135-163, 2003.

[18] D. Mukherjee, "Effect of diffusion on a two-species ecoepidemiological model," Mathematical and Computer Modelling of Dynamcial Systems, vol. 11, no. 4, pp. 447-457, 2005.

[19] J. F. Moxnes and K. Hausken, "Mathematical modelling of acute virus influenza A infections," Mathematical and Computer Modelling of Dynamical Systems, vol. 18, no. 5, pp. 521-538, 2012.

[20] L. Liu, J. Wu, and X.-Q. Zhao, "The impact of migrant workers on the tuberculosis transmission: general models and a case study for China," Mathematical Biosciences and Engineering, vol. 9, no. 4, pp. 785-807, 2012.

[21] Y. Lou and X.-Q. Zhao, "Modelling malaria control by introduction of larvivorous fish," Bulletin of Mathematical Biology, vol. 73, no. 10, pp. 2384-2407, 2011.

[22] K. A. Pawelek, S. Liu, F. Pahlevani, and L. Rong, "A model of HIV-1 infection with two time delays: mathematical analysis and comparison with patient data," Mathematical Biosciences, vol. 235, no. 1, pp. 98-109, 2012.

[23] X. Wang, Y. Tao, and X. Song, "Pulse vaccination on SEIR epidemic model with nonlinear incidence rate," Applied Mathematics and Computation, vol. 210, no. 2, pp. 398-404, 2009.

[24] W. Ma, M. Song, and Y. Takeuchi, "Global stability of an SIR epidemic model with time delay," Applied Mathematics Letters, vol. 17, no. 10, pp. 1141-1145, 2004.

[25] W. Wang and X.-Q. Zhao, "Basic reproduction numbers for reaction-diffusion epidemic models," SIAM Journal on Applied Dynamical Systems, vol. 11, no. 4, pp. 1652-1673, 2012. 
[26] P. van den Driessche and X. Zou, "Global attractivity in delayed Hopfield neural network models," SIAM Journal on Applied Mathematics, vol. 58, no. 6, pp. 1878-1890, 1998.

[27] M. Y. Li and H. Shu, "Impact of intracellular delays and targetcell dynamics on in vivo viral infections," SIAM Journal on Applied Mathematics, vol. 70, no. 7, pp. 2434-2448, 2010.

[28] H. Guo, M. Y. Li, and Z. Shuai, "Global stability of the endemic equilibrium of multigroup SIR epidemic models," Canadian Applied Mathematics Quarterly, vol. 14, no. 3, pp. 259-284, 2006.

[29] J. Wang, S. Liu, and Y. Takeuchi, "Threshold dynamics in a periodic SVEIR epidemic model," International Journal of Biomathematics, vol. 4, no. 4, pp. 493-509, 2011.

[30] S. Liu and L. Wang, "Global stability of an HIV-1 model with distributed intracellular delays and a combination therapy," Mathematical Biosciences and Engineering, vol. 7, no. 3, pp. 675685, 2010.

[31] G. Huang, Y. Takeuchi, and W. Ma, "Lyapunov functionals for delay differential equations model of viral infections," SIAM Journal on Applied Mathematics, vol. 70, no. 7, pp. 2693-2708, 2010.

[32] K. Hattaf, A. A. Lashari, Y. Louartassi, and N. Yousfi, "A delayed SIR epidemic model with general incidence rate," Electronic Journal of Qualitative Theory of Differential Equations, vol. 2013, no. 3, pp. 1-9, 2013.

[33] X. Wang and S. Liu, "Global properties of a delayed SIR epidemic model with multiple parallel infectious stages," Mathematical Biosciences and Engineering, vol. 9, no. 3, pp. 685-695, 2012.

[34] J. Wei and S. Ruan, "Stability and bifurcation in a neural network model with two delays," Physica D, vol. 130, no. 3-4, pp. 255-272, 1999.

[35] S. Ruan and J. Wei, "On the zeros of transcendental functions with applications to stability of delay differential equations with two delays," Dynamics of Continuous, Discrete \& Impulsive Systems. Series A, vol. 10, no. 6, pp. 863-874, 2003.

[36] M. Fan and K. Wang, "Periodic solutions of a discrete time nonautonomous ratio-dependent predator-prey system," Mathematical and Computer Modelling, vol. 35, no. 9-10, pp. 951-961, 2002.

[37] H. Wang and W. Jiang, "Hopf-pitchfork bifurcation in van der Pol's oscillator with nonlinear delayed feedback," Journal of Mathematical Analysis and Applications, vol. 368, no. 1, pp. 9$18,2010$.

[38] W. Jiang and Y. Yuan, "Bogdanov-Takens singularity in van der Pol's oscillator with delayed feedback," Physica D, vol. 227, no. 2, pp. 149-161, 2007.

[39] Z. Chen, "Periodic solutions for Nicholson-type delay system with nonlinear density-dependent mortality terms," Electronic Journal of Qualitative Theory of Differential Equations, vol. 2013, no. 1, pp. 1-10, 2013.

[40] C. Zhang, Y. Zhang, and B. Zheng, "A model in a coupled system of simple neural oscillators with delays," Journal of Computational and Applied Mathematics, vol. 229, no. 1, pp. 264-273, 2009.

[41] S. Guo, Y. Chen, and J. Wu, "Two-parameter bifurcations in a network of two neurons with multiple delays," Journal of Differential Equations, vol. 244, no. 2, pp. 444-486, 2008.

[42] B. Niu and J. Wei, "Stability and bifurcation analysis in an amplitude equation with delayed feedback," Chaos, Solitons \& Fractals, vol. 37, no. 5, pp. 1362-1371, 2008.
[43] S. Ma, Q. Lu, and Z. Feng, "Double Hopf bifurcation for van der Pol-Duffing oscillator with parametric delay feedback control," Journal of Mathematical Analysis and Applications, vol. 338, no. 2, pp. 993-1007, 2008.

[44] Y. Ding, W. Jiang, and P. Yu, "Double Hopf bifurcation in a container crane model with delayed position feedback," Applied Mathematics and Computation, vol. 219, no. 17, pp. 9270-9281, 2013.

[45] J. Hale and S. Lunel, Functional Differential Equations, vol. 99 of Applied Mathematical Sciences, Springer, New York, NY, USA, 1993.

[46] B. D. Hassard, N. D. Kazarinoff, and Y. H. Wan, Theory and Applications of Hopf Bifurcation, vol. 41 of London Mathematical Society Lecture Note Series, Cambridge University Press, Cambridge, Mass, USA, 1981.

[47] L. Wen and X. Yang, "Global stability of a delayed SIRS model with temporary immunity," Chaos, Solitons \& Fractals, vol. 38, no. 1, pp. 221-226, 2008.

[48] J. P. LaSalle, The Stability of Dynamical Systems, Regional Conference Series in Applied Mathematics, SIAM, Philadelphia, Pa, USA, 1976. 


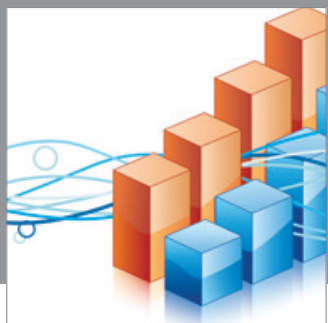

Advances in

Operations Research

mansans

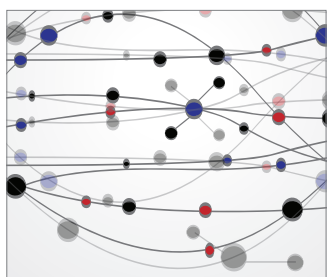

The Scientific World Journal
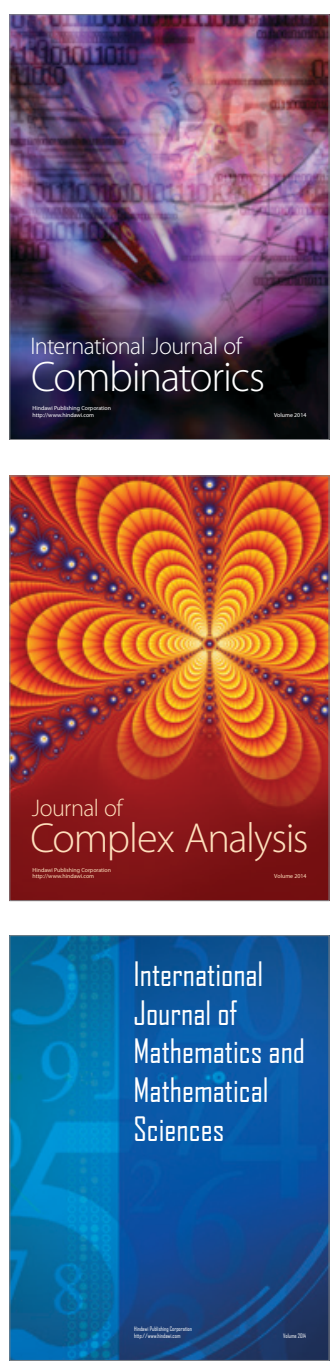
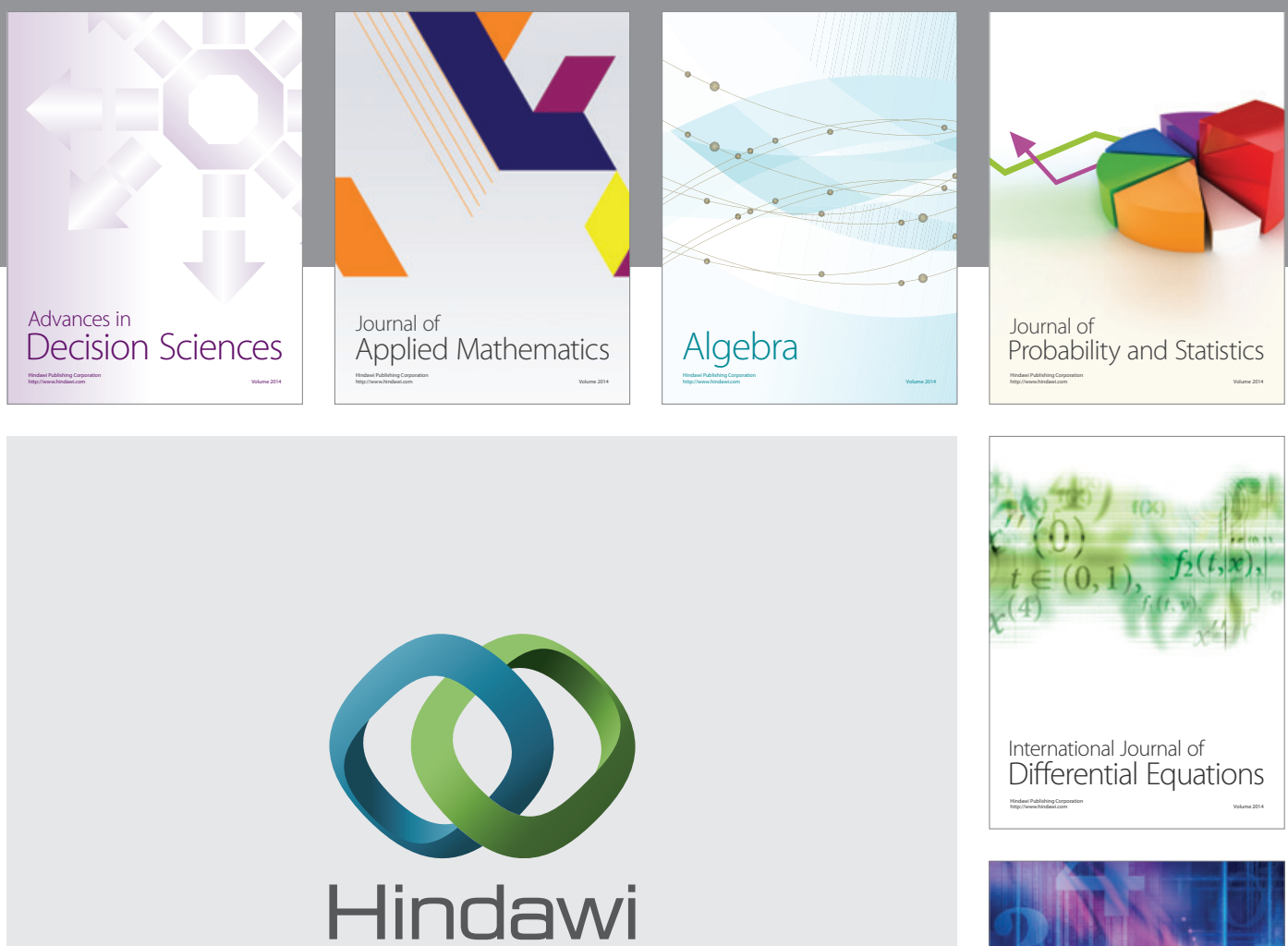

Submit your manuscripts at http://www.hindawi.com
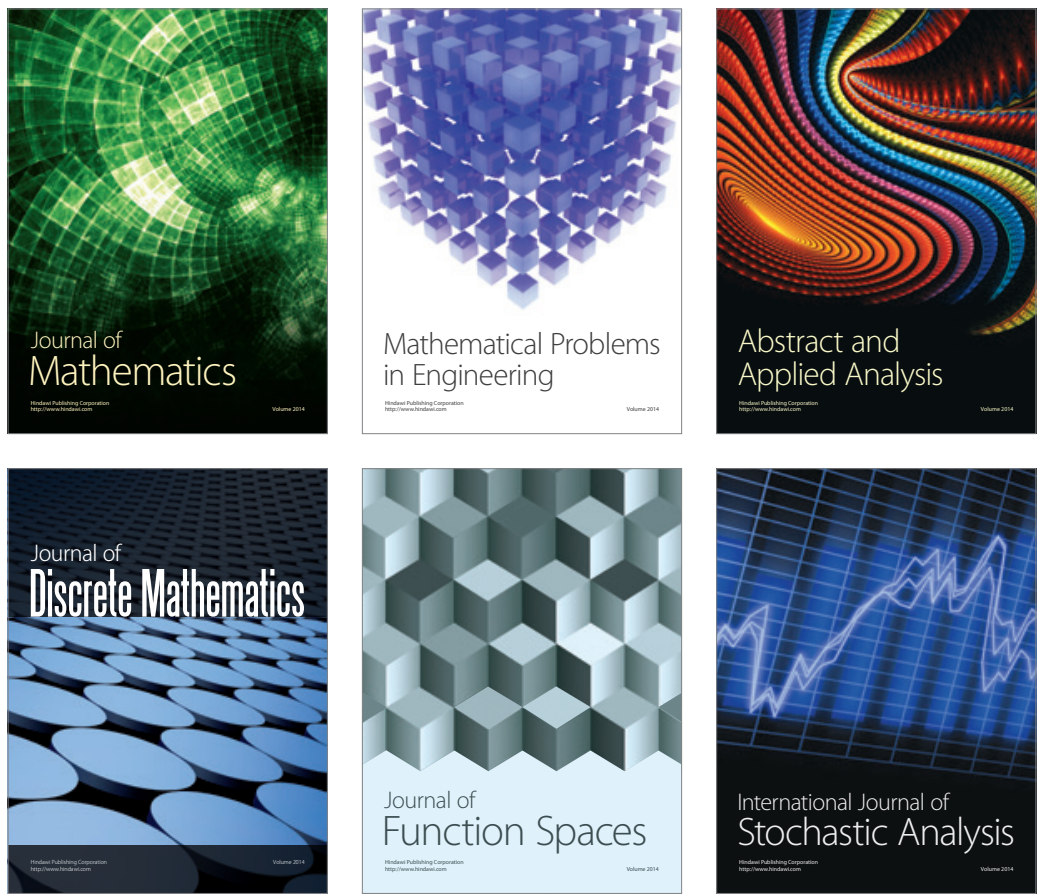

Journal of

Function Spaces

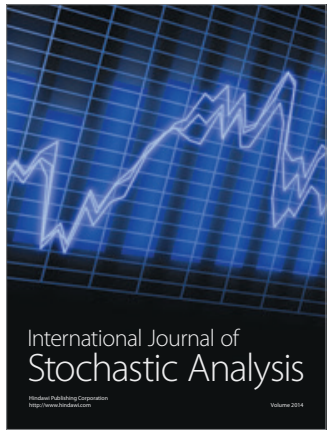

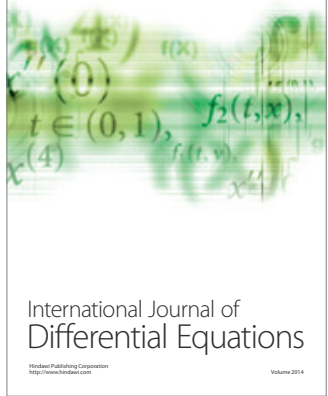
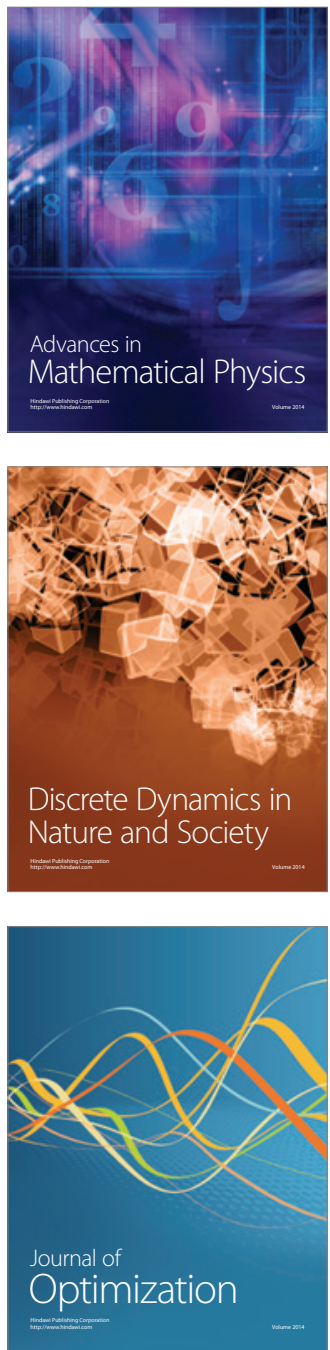